PROCEEDINGS OF THE

AMERICAN MATHEMATICAL SOCIETY

Volume 125, Number 6, June 1997, Pages 1751-1754

S 0002-9939(97)03813-6

\title{
A NOTE ON THE PATH HOLOMORPHY OF RANDOM FUNCTIONS HOLOMORPHIC IN MEAN
}

\author{
F. MARTINEZ AND A. R. VILLENA
}

(Communicated by Palle E. T. Jorgensen)

\begin{abstract}
Every random function holomorphic in mean on an open subset of the complex field is equivalent to a random function with almost all its paths holomorphic on $D$.
\end{abstract}

Throughout the paper, $(\Omega, \Sigma, \mathbb{P})$ denotes a complete probability space and $D$ an open subset of the complex field.

A map $f: D \times \Omega \rightarrow \mathbb{C}$ is said to be a (first-order) random function on $D$ if, for each $z \in D$, the map $\omega \mapsto f(z, \omega)$ lies in $\mathcal{L}_{1}(\mathbb{P})$, the linear space of all first-order random variables. For every fixed $\omega \in \Omega$, the function $z \mapsto f(z, \omega)$ from $D$ into $\mathbb{C}$ is called a path of $f$. Two random functions $f$ and $g$ on $D$ are said to be equivalent, and we denote it by $f \equiv g$, if $f(z, \omega)=g(z, \omega)$ almost surely for every $z \in D$. Given $\xi \in \mathcal{L}_{1}(\mathbb{P}),[\xi]$ denotes the equivalence class of $\xi$ for the usual almost surely identification. The space $L_{1}(\mathbb{P})=\left\{[\xi]: \xi \in \mathcal{L}_{1}(\mathbb{P})\right\}$ becomes a complex Banach space with the norm $\|[\xi]\|_{1}=\int_{\Omega}|\xi| d \mathbb{P}$.

A random function $f$ on $D$ is said to be holomorphic in mean on $D$ if, for every $z_{0} \in D$, the quotient $\frac{f(z, \cdot)-f\left(z_{0}, \cdot\right)}{z-z_{0}}$ has a limit in mean as $z$ approaches $z_{0}$, equivalently the function $z \mapsto[f(z, \cdot)]$ from $D$ into the complex Banach space $L_{1}(\mathbb{P})$ is holomorphic in the traditional sense. For a full discussion of holomorphic vectorvalued functions the reader is referred to [1, Section 3.2]. It should be pointed out that a random function $f$ is holomorphic in mean on $D$ if, and only if, for every $\xi \in \mathcal{L}_{\infty}(\mathbb{P})$ the complex valued function $z \mapsto \int_{\Omega} f(z, \omega) \xi(\omega) d \mathbb{P}$ is holomorphic on $D$, where $\mathcal{L}_{\infty}(\mathbb{P})$ is the linear space of all essentially bounded random variables (see [1, Definition 3.10.1 and Proposition 3.10.1]).

The purpose of this paper is to prove the following surprising result.

Theorem. Let $f$ be a random function on D. Then the following conditions are equivalent:

1. $f$ is holomorphic in mean on $D$.

2. $f$ is equivalent to a random function $g$ continuous in mean with almost all its paths holomorphic on $D$.

We note that there are random functions holomorphic in mean which have no holomorphic paths (see Example 1) and we emphasize that there exists a random function $f$ continuous in mean, but such that there is no random function $g$ that is equivalent to $f$ and has a non-negligible set of continuous paths (see Example 2).

Received by the editors December 11, 1995.

1991 Mathematics Subject Classification. Primary 30G30, 60G17.

(C)1997 American Mathematical Society 
Example 1. Let $D=\{x+i y \in \mathbb{C}: 0<x, y<1\}$ and consider $\Omega$ as $D$ endowed with the Lebesgue measure. The function $f: D \times \Omega \rightarrow \mathbb{C}$ given by $f(z, \omega)=1$ if $z=\omega$ and $f(z, \omega)=0$ otherwise, is holomorphic in mean on $D$ and has no holomorphic paths.

Example 2. Consider the interval $[0,1]$ endowed with the Lebesgue measure and the sequence $\left\{\xi_{n}\right\}$ of random variables given by $\xi_{n}(\omega)=1$ if $2^{-k} j \leq \omega \leq 2^{-k}(j+1)$ and $\xi_{n}(\omega)=0$ otherwise, where $n=2^{k}+j, 0 \leq k, 0 \leq j<2^{k}$. Define the random function $f$ on the open unit disc $D$ by $f(z, \omega)=n^{2}(n+1)^{2}\left(\frac{1}{n}-|z|\right)\left(|z|-\frac{1}{n+1}\right) \xi_{n}(\omega)$ if $\frac{1}{n+1} \leq|z| \leq \frac{1}{n}, n \in \mathbb{N}$, and $f(0, \omega)=0$, which is obviously continuous in mean on $D \backslash\{0\}$. Given a sequence $\left\{z_{n}\right\}$ in $D \backslash\{0\}$ converging to zero we have $\int_{0}^{1}\left|f\left(z_{n}, \omega\right)\right| d \omega \leq \frac{1}{4}\left\|\xi_{k_{n}}\right\|_{1}$, where $k_{n}$ satisfies $\frac{1}{k_{n}+1} \leq\left|z_{n}\right| \leq \frac{1}{k_{n}}$. Since $\left\{\left\|\xi_{k_{n}}\right\|_{1}\right\}$ converges to zero we conclude that $f$ is continuous in mean at zero. Now we note that $f\left(\frac{2 n+1}{2 n(n+1)}, \omega\right)=\frac{1}{4} \xi_{n}(\omega)$ for all $n \in \mathbb{N}$ and $\omega \in[0,1]$. Since the sequence $\left\{\xi_{n}\right\}$ converges nowhere on $[0,1]$ it may be concluded that almost every path of any random function equivalent to $f$ is not continuous at zero.

Given a random function continuous in mean on $D$ and $\gamma$ a piecewise smooth curve in $D$, parametrized say in the interval $[\alpha, \beta]$, the function $t \mapsto[f(\gamma(t), \cdot)] \gamma^{\prime}(t)$ from $[\alpha, \beta]$ into $L_{1}(\mathbb{P})$ is Riemann integrable. Let us denote $\int_{\gamma}[f(z, \cdot)] d z=$ $\int_{\alpha}^{\beta}[f(\gamma(t), \cdot)] \gamma^{\prime}(t)$.

Lemma 1. Let $f$ be a random function continuous in mean on $D$ and assume that almost all its paths are continuous on D. If $\gamma$ is a piecewise smooth curve in $D$, then the function $\omega \mapsto \int_{\gamma} f(z, \omega) d z$ lies in the equivalence class $\int_{\gamma}[f(z, \cdot)] d z$.

Proof. There is no loss of generality in assuming $\gamma$ parametrized in the interval $[0,1]$.

By [2, Proposition 2.7.12] the sequence $\left\{\left[\frac{1}{n} \sum_{j=1}^{n} f\left(\gamma\left(\frac{j}{n}\right), \cdot\right) \gamma^{\prime}\left(\frac{j}{n}\right)\right]\right\}$ converges in mean to $\int_{\gamma}[f(z, \cdot)] d z$. From [2, Proposition 2.3.10] it follows that there exists a strictly increasing sequence $\left\{n_{k}\right\}$ of natural numbers such that the sequence $\left\{\frac{1}{n_{k}} \sum_{j=1}^{n_{k}} f\left(\gamma\left(\frac{j}{n_{k}}\right), \cdot\right) \gamma^{\prime}\left(\frac{j}{n_{k}}\right)\right\}$ converges almost surely to a function in $\mathcal{L}_{1}(\mathbb{P})$. We note that, for almost every $\omega \in \Omega,\left\{\frac{1}{n_{k}} \sum_{j=1}^{n_{k}} f\left(\gamma\left(\frac{j}{n_{k}}\right), \omega\right) \gamma^{\prime}\left(\frac{j}{n_{k}}\right)\right\}$ converges to $\int_{\gamma} f(z, \omega) d z$ and therefore, from [2, Corollary 2.3.12], it may be concluded that the function $\omega \mapsto \int_{\gamma} f(z, \omega) d z$ lies in $\int_{\gamma}[f(z, \cdot)] d z$.

Lemma 2. Let $\sum \xi_{n}\left(z-z_{0}\right)^{n}$ a power series with first-order random coefficients and a non-zero radius of convergence in mean, say $R$. Then there exists a negligible set $\Delta$ such that, for each $\omega \in \Omega \backslash \Delta$, the radius of convergence of the power series $\sum \xi_{n}(\omega)\left(z-z_{0}\right)^{n}$ is at least $R$.

Proof. Consider $0<r<R$. By [1, Theorem 3.11.4] the series $\sum \int_{\Omega}\left|\xi_{n}\right| r^{n} d \mathbb{P}$ converges and therefore [2, Proposition 2.5.1] there exists a negligible set $\Delta_{r}$ such that, for every $\omega \in \Omega \backslash \Delta_{r}$, the series $\sum \xi_{n}(\omega) r^{n}$ converges (absolutely) to a function in $\mathcal{L}_{1}(\mathbb{P})$. Moreover, for all $\omega \in \Omega \backslash \Delta_{r}$ and $\left|z-z_{0}\right| \leq r$ the series $\sum \xi_{n}(\omega)\left(z-z_{0}\right)^{n}$ converges. Choose a sequence $\left\{r_{n}\right\} \rightarrow R$ with $0<r_{n}<R \forall n \in \mathbb{N}$ and let us denote by $\Delta$ the negligible set given by $\Delta=\bigcup_{n=1}^{\infty} \Delta_{r_{n}}$. It is a simple matter to show that, for all $\omega \in \Omega \backslash \Delta$ and $\left|z-z_{0}\right|<R$ the series $\sum \xi_{n}(\omega)\left(z-z_{0}\right)^{n}$ converges, which completes the proof. 
Lemma 3. Let $f$ and $g$ be random functions holomorphic in mean on $D$ and assume that $f$ and $g$ have almost all its paths holomorphic on $D$. If $f \equiv g$, then $\mathbb{P}[f(\cdot, \omega)=g(\cdot, \omega)]=1$.

Proof. Let $\Lambda \in \Sigma$ with $\mathbb{P}[\Lambda]=1$ and such that the paths $f(\cdot, \omega)$ and $g(\cdot, \omega)$ are holomorphic on $D$ whenever $\omega$ lies in $\Lambda$.

Write $D=\bigcup_{n=1}^{\infty} D_{n}$ for a sequence $\left\{D_{n}\right\}$ of pairwise disjoint connected open subsets. Fix $n \in \mathbb{N}$ and let $\Delta_{n}=\left\{\omega \in \Omega: f(z, \omega)=g(z, \omega) \forall z \in D_{n}\right\}$. Choose a sequence $\left\{z_{k}\right\}$ in $D_{n}$ converging to $z_{0} \in D$. From the uniqueness theorem [1, Theorem 3.11.5] it follows that, for a given $\omega \in \Lambda, f(z, \omega)=g(z, \omega) \forall z \in D_{n}$ if, and only if, $f\left(z_{k}, \omega\right)=g\left(z_{k}, \omega\right) \forall k \in \mathbb{N} \cup\{0\}$. Thus we have

$$
\Delta_{n} \cap \Lambda=\bigcap_{k=0}^{\infty}\left\{\omega \in \Lambda: f\left(z_{k}, \omega\right)=g\left(z_{k}, \omega\right)\right\},
$$

which proves that $\Delta_{n}$ is measurable with $\mathbb{P}\left[\Delta_{n}\right]=1$. Therefore $\mathbb{P}[f(\cdot, \omega)=$ $g(\cdot, \omega)]=1$, since $\{\omega \in \Omega: f(\cdot, \omega)=g(\cdot, \omega)\}=\bigcap_{n=1}^{\infty} \Delta_{n}$.

Proof of the Theorem. Assume the assertion 1 holds. If $D=\mathbb{C}$, then $[f(z, \cdot)]$ can be expanded on $\mathbb{C}$ as the sum in mean of a power series $\sum\left[\xi_{n}\right] z^{n}$ with $\xi_{n} \in \mathcal{L}_{1}(\mathbb{P}) \forall n \in$ $\mathbb{N} \cup\{0\}$. The series $\sum \xi_{n} z^{n}$ satisfies the requirements in Lemma 2. If $\Delta$ is the negligible set given by that lemma, then we define $g(z, \omega)=\sum_{n=0}^{\infty} \xi_{n}(\omega) z^{n} \forall(z, \omega) \in$ $\mathbb{C} \times(\Omega \backslash \Delta)$ and $g(z, \omega)=0$ otherwise. The function $g$ clearly satisfies our requirements in assertion 2. If $D \neq \mathbb{C}$, then we consider the countable set $B=$ $\{p+q i \in D: p, q \in \mathbb{Q}\}$. For each $b \in B$, set $r_{b}=\inf \{|z-b|: z \notin D\}$ and write $D_{b}=\left\{z \in \mathbb{C}:|z-b|<r_{b}\right\}$. For every $b \in B$ we can write $[f(z, \cdot)]$ on $D_{b}$ as the sum in mean of a power series $\sum\left[\xi_{n}^{(b)}\right](z-b)^{n}$ with $\xi_{n}^{(b)} \in \mathcal{L}_{1}(\mathbb{P}) \forall n \in \mathbb{N} \cup\{0\}$. The series $\sum \xi_{n}^{(b)}(z-b)^{n}$ satisfies the requirements in Lemma 2. Let us denote by $\Delta_{b}$ the negligible set given by that lemma and let $g_{b}(z, \omega)=\sum \xi_{n}^{(b)}(\omega)(z-b)^{n} \forall(z, \omega) \in$ $D_{b} \times\left(\Omega \backslash \Delta_{b}\right)$ and $g(z, \omega)=0$ otherwise. Note that $g_{b}$ is holomorphic in mean on $D_{b}$ and all its paths are holomorphic on $D_{b}$. Given $b, c \in B$ with $D_{b} \cap D_{c} \neq \varnothing, g_{b}$ and $g_{c}$ are clearly equivalent on $D_{b} \cap D_{c}$ and Lemma 3 gives a negligible set $\Delta_{b, c}$ such that $g_{b}(\cdot, \omega)=g_{c}(\cdot, \omega) \forall \omega \in \Omega \backslash \Delta_{b, c}$. Finally we take $\Delta=\bigcup_{b, c \in B} \Delta_{b, c}$ and we define the function $g$ on $D \times \Omega$ by $g(z, \omega)=g_{b}(z, \omega)$ if $(z, \omega) \in D_{b} \times \Omega \backslash \Delta$ and $g(z, \omega)=0$ otherwise. Then $g$ is a well-defined first-order random function on $D$ with holomorphic paths. Moreover, it is clear that $f \equiv g$.

Conversely, suppose that assertion 2 holds. Note that $f$ is continuous in mean on $D$. In order to prove the holomorphy in mean of $f$ we can assume that $D$ is an open disc. Consider $\xi \in \mathcal{L}_{\infty}(\mathbb{P})$. For all $z, w \in D$ we have

$$
\left|\int_{\Omega} f(z, \omega) \xi(\omega) d \mathbb{P}-\int_{\Omega} f(w, \omega) \xi(\omega) d \mathbb{P}\right| \leq\|\xi\|_{\infty}\|f(z, \cdot)-f(w, \cdot)\|_{1},
$$

which shows the continuity on $D$ of the function $\int_{\Omega} f(\cdot, \omega) \xi(\omega) d \mathbb{P}$. Let $\gamma$ be a piecewise smooth closed curve in $D$. Then $\int_{\gamma} g(z, \omega) d z=0$ for almost every $\omega \in \Omega$, since almost every path function of $g$ is holomorphic on $D$. From Lemma 1 it follows that $\int_{\gamma}[g(z, \cdot)] d z=0$ and finally we note that $\int_{\gamma}[f(z, \cdot)] d z=\int_{\gamma}[g(z, \cdot)] d z$. On account of the continuity of the linear functional $\zeta \mapsto \int_{\Omega} \zeta \xi d \mathbb{P}$ on $L_{1}(\mathbb{P}),[2$, Proposition 2.3.7(5)] shows that

$$
0=\int_{\Omega}\left(\int_{\gamma}[f(z, \cdot)] d z\right) \xi d \mathbb{P}=\int_{\gamma}\left(\int_{\Omega} f(z, \omega) \xi(\omega) d \mathbb{P}\right) d z .
$$


According to Morera's Theorem, every function $\int_{\Omega} f(\cdot, \omega) \xi(\omega) d \mathbb{P}$ is holomorphic on $D$ and we conclude that $f$ is holomorphic in mean on $D$.

\section{REFERENCES}

[1] E. Hille and R. S. Phillips, Functional analysis and semigroups, Amer. Math. Soc. Coll. 31, Providence, 1957. MR 19:664d

[2] C. M. Marle, Measures et probabilités, Hermann, Paris, 1974.

Departamento de Estadística e I. O., Facultad de Ciencias, Universidad de Granada, 18071 Granada, Spain

Departamento de Análisis Matemático, Facultad de Ciencias, Universidad de Granada, 18071 Granada, Spain

E-mail address: avillena@goliat.ugr.es 